\title{
Advances in the prevention, management, and treatment of community-acquired pneumonia Mathias W Pletz ${ }^{1}$, Tobias Welte ${ }^{1}$ and Sebastian $\mathrm{R} \mathrm{Ott}^{2}$
}

\author{
Addresses: ${ }^{1}$ Department of Pulmonary Medicine, Hannover Medical School, Carl-Neuberg-Strasse 1, Hannover, D-30625, Germany; \\ ${ }^{2}$ Department of Pulmonary Medicine, Inselspital, University Hospital and University of Bern, Bern, CH-3010, Switzerland \\ *Corresponding author: Mathias W Pletz (pletz.mathias@mh-hannover.de) \\ Fl000 Medicine Reports 2010, 2:53 (doi:10.3410/M2-53)
}

The electronic version of this article is the complete one and can be found at: http://fl000.com/reports/medicine/content/2/53

\begin{abstract}
Despite the availability of powerful antibiotics, community-acquired pneumonia (CAP) remains one of the leading reasons for morbidity and mortality worldwide, and despite the availability of powerful antibiotics, there has been only little improvement in case fatality rates for many years. Consequently, it cannot be expected that novel antibiotics will substantially improve outcomes in CAP. Therefore, this review focuses on novel approaches that may reduce CAP-related mortality: the impact of immunomodulation by macrolides and fluoroquinolones and the prevention of CAP by pneumococcal vaccines.
\end{abstract}

\section{Introduction and context}

Despite the availability of powerful antibiotics, community-acquired pneumonia (CAP) remains one of the leading reasons for morbidity and mortality worldwide. Case fatality rates vary widely among patients with CAP. Recent data of the German CAPNETZ (competence network for community-acquired pneumonia) show that fatality rates were $0.5 \%$ for outpatients and $14 \%$ for hospitalized patients [1]. Owing to insufficient diagnostic tools, pathogens are detected in only $30-50 \%$ of hospitalized patients with CAP. In most studies, Streptococcus pneumoniae is by far the leading pathogen in CAP. A study using transthoracic needle aspiration showed that $S$. pneumoniae is found frequently when sputum and blood culture fail to detect any pathogen [2].

During the last decade, the case fatality rate of patients hospitalized with CAP has not really changed. Therefore, novel approaches are needed to further improve outcome in hospitalized patients with CAP. Recent advances in CAP include the recognition of antiinflammatory strategies and prevention of CAP by vaccination.

\section{Recent advances Treatment}

The primary rationale for the use of antibiotics in the treatment of CAP is undoubtedly their direct antimicrobial effect. But in the last two decades, growing evidence has shown that at least some classes of antibiotics possess beneficial properties that go beyond just killing of the causative agent; they appear to modulate the host's immune response. The best known and investigated are the immunomodulatory effects of macrolides and to a certain degree the immunorelevant actions of quinolones.

Immunomodulatory properties of antibiotics were recognized firstly in macrolides more than 20 years ago, when the 5-year survival rate of patients with diffuse panbronchiolitis (DPB) could be improved from less than $50 \%$ (approximately $8 \%$ if the patient was chronically colonized with Pseudomonas aeruginosa) to approximately $90 \%$ by long-term administration of erythromycin, despite macrolide resistance of the involved pseudomonal strains and application in subantimicrobial doses $[3,4]$. Subsequently, great efforts have been taken to elucidate the underlying mechanisms 
and have revealed interesting insights into the interference of antibiotic agents with neutrophil activation and mobilization, neutrophil apoptosis, activation of nuclear transcription factors, production of reactive oxygen species, airway mucus secretion, and bacterial bio-film [5]. Specifically, macrolides possess the ability to suppress the production and secretion of proinflammatory cytokines from monocytes and normal human bronchial epithelial cells through the inhibition of extracellular signal-regulated kinases (ERKs). Owing in part to direct and indirect downregulation of nuclear factor-kappa-B (NF- $\kappa \mathrm{B})$, this leads to decreased levels of interleukin (IL)-8, tumor necrosis factor-alpha (TNF- $\alpha$ ), granulocyte-macrophage colony-stimulating factor, and IL-1 $\beta[6,7]$. As a consequence of lower levels of IL- 8 and other chemo-attractants and adhesion molecules (e.g., E-selectin and intercellular adhesion molecule-1), macrolides inhibit the recruitment and accumulation of neutrophil granulocytes at the site of inflammation and thus prevent the release of lysosomal enzymes and generation of reactive oxygen species and the resulting airway damage. This effect was confirmed recently by Bosnar et al. [8], who demonstrated that pre-treatment with azithromycin and clarithromycin prior to lipoploysaccharide (LPS) challenge in mice can significantly reduce total cell and neutrophil numbers in bronchoalveolar lavage fluid (BALF) and myeloperoxidase concentration in lung tissue.

Respiratory quinolones have also been shown to impair macrophage chemotaxis in rats and to significantly reduce transendothelial neutrophil and monocyte migration $[9,10]$. In addition, Weiss et al. [11] demonstrated that pre-incubation of LPS-activated human monocytes with moxifloxacin causes a concentration-dependent inhibition of the synthesis of IL-1 $\beta$, IL-8, and TNF- $\alpha$. Preincubation with moxifloxacin was also associated with reduced expressions of NF- $\kappa \mathrm{B}, \mathrm{ERK}$, and c-Jun N-terminal kinase [11]. Recently, Ogino et al. [12] confirmed the inhibitory effect of different fluoroquinolones on LPSinduced pro-inflammatory cytokine production (IL-1 $\beta$ and TNF- $\alpha$ ) in murine in vivo and in vitro experiments. In addition, they found that this effect can be attributed in particular to those fluoroquinolones with a cyclopropyl group at the N1 position (e.g., ciprofloxacin, moxifloxacin, grepafloxacin, and sparfloxacin).

Whereas the beneficial effects of macrolides in chronic pulmonary inflammatory conditions like DPB, cystic fibrosis, or bronchiolitis obliterans syndrome after lung transplantation are widely accepted [13], their impact on acute pulmonary inflammation is currently under intensive investigation. Experimental studies investigating the immunomodulatory actions of macrolides and ketolides in murine models of acute pneumonia caused by Mycoplasma pneumonia and Chlamydia pneumophilia showed decreased concentrations of various pro-inflammatory cytokines (e.g., IL-1 $\beta$, IL-8, and TNF- $\alpha$ ) in BALF and reduced lung histological signs of inflammation, although complete bacterial eradication could not be achieved in all cases [14-16]. Therefore, it might be concluded that macrolides provide immunomodulatory effects in acute inflammation.

The first clinical evidence for the probability of immunomodulatory effects of macrolides, even in acute inflammation, was derived mainly from retrospective cohort studies evaluating the impact of an additional macrolide treatment in patients with CAP [17-19]. These studies could demonstrate that the addition of a macrolide to a beta-lactam significantly decreased the risk of death. This effect was also seen in severely ill patients with severe sepsis due to pneumonia [20]. Tessmer et al. [21] recently evaluated the benefit of beta-lactam/macrolide combination therapy (BLM) versus beta-lactam monotherapy in a prospective cohort study that included hospitalized patients with CAP. The authors could clearly demonstrate a significantly reduced adjusted mortality rate in patients with severe CAP (confusion, respiratory rate, blood pressure, and age [whether below or above 65 years old] score [CRB65] of at least 2) receiving BLM therapy. Interestingly, this effect was not seen in mild to moderate pneumonia (CRB65 of not more than 1). Finch et al. [22] demonstrated a similar clinical effect for quinolones.

In conclusion, there is strong evidence of an immunomodulatory effect of macrolides and some evidence of an immunomodulatory effect of quinolones in acute pulmonary inflammation and a subsequent clinical benefit in terms of improved outcome and survival in CAP. Unfortunately, most data derive from retrospective or non-randomized prospective trials. Hence, before the potential advantage of macrolides and quinolones in the treatment of CAP can be generally stated, prospective randomized controlled studies are required.

Statins are considered to have anti-inflammatory effects and prior use of statins has been linked to improved outcome of CAP. In a monocenter prospective observational study, Chalmers and colleagues [23] found that patients on statins had a higher severity of disease according to the pneumonia severity index but a decreased 30-day mortality (adjusted odds ratio 0.46). As a marker of inflammation, C-reactive protein was lower and decreased earlier in these patients. The authors controlled for the so-called 'healthy-user effect' by demonstrating that other drugs prescribed for cardiovascular disease had no influence on the outcome of CAP. 
Another recent trial could not confirm a beneficial effect of prior statin use on the risk of CAP in a populationbased cohort of older individuals (more than 65 years) [24]. In this case control study, inhabitants of nursing homes were excluded because they are more likely to suffer from certain severe comorbid illnesses, frailty, and cognitive or functional impairment and thus are at increased risk of pneumonia. Despite the increased risk of $\mathrm{CAP}$, frail older people or those with more severe comorbidities are less likely to receive statins [25]. In addition, subjects receiving statins tend to practice other healthy behaviors, like physical activity or smoking cessation, and are more likely to have better functional status and comorbidity that is less severe. Hence, the authors postulate that a 'healthy-user bias' is responsible for the decreased CAP risk that has been described in earlier studies.

Since the two studies had different endpoints (outcome of CAP [23] versus risk of CAP [24]), it is difficult to draw any final conclusion from their findings. Therefore, the results of prospective randomized controlled trials that address the immunomodulatory properties of statins and that are currently under way should be awaited before allowing any definitive recommendation for clinical practice.

Whereas systemic steroids appear to be beneficial in patients with vasopressor-dependent septic shock and in patients with early severe acute respiratory distress syndrome (especially when critical illness-related corticosteroid insufficiency is present), their role as adjunctive treatment in patients with CAP remains controversial [26-30]. Confalonieri et al. [27] demonstrated that lowdose glucocorticoid infusion may have beneficial effects on organ dysfunction in severe pneumonia. Owing to the small sample size and some baseline imbalances, this study should be valued as a hypothesis-generating study rather than as a set of definitive results. In contrast to this study and other trials suggesting that adjunctive steroid treatment is advantageous in CAP [28], a recent randomized, placebo-controlled study could not confirm any improved CAP outcome attributed to steroid treatment [29].

Although adjunctive treatment with glucocorticoids in severe pneumonia is probably indicated, further randomized clinical trials are urgently needed to confirm the preliminary positive results and to define optimal dosage and duration of this treatment. A large (inclusion of more than 1000 patients is planned) randomized trial that was initiated by the Veteran Administration Cooperative Study Group and that will soon be under way in the US will probably define the significance of steroid application in CAP treatment.

\section{Prevention}

Recent advances in the prevention of CAP include two dynamic fields: vaccination (i.e., pneumococcal and influenza vaccination) and the recognition of the influence of prior medication and conditions (e.g., inhaled corticosteroids and smoking).

It has taken many years to successfully develop an efficacious vaccine against $S$. pneumoniae. The main reason is the low immunogenicity of polysaccharides, which are the target of opsonizing antibodies. Two types of vaccines are currently in clinical use.

One type of vaccine contains purified capsular polysaccharides from 23 of the known 91 pneumococcal serotypes (pneumococcal polysaccharides vaccine [PPV]-23). Polysaccharides induce primarily a B celldependent immune response and are not effective in children younger than 2 years of age (probably due to their immature immune system). The polysaccharide vaccine is known to reduce the rate of bacteremia, but numerous studies and a meta-analysis have demonstrated little or no effect on the prevention of pneumococcal pneumonia, particularly in immunosenescent or immunosuppressed patients [31]. However, a recent prospective, multicenter, randomized clinical trial showed reduction of pneumococcal pneumonia by $64 \%$ in Japanese nursing home residents and significantly reduced mortality from pneumococcal pneumonia in patients vaccinated with PPV-23 [32]. In a US cohort study analyzing over 60,000 patients with HIV, subjects who received PPV-23 had a lower rate of pneumonia (incidence rate ratio 0.8, 95\% confidence interval 0.8-0.9) than patients who had never been vaccinated, independently of recent CD4 count, HIV viral load, antiretroviral therapy, and history of pneumonia [33]. In conclusion, results regarding the efficacy of PPV-23 in pneumonia remain controversial.

Pneumococcal conjugate vaccine (PCV) was designed for children younger than 2 years and initially contained capsular polysaccharides from seven serotypes. Capsular polysaccharides of PCV are conjugated to highly immunogenic proteins, resulting in B- and T-cell response with consequent mucosal immunity. Studies have shown that vaccination reduces the carrier rate for serotypes contained in the vaccine [34]. Since children are the main reservoir of $S$. pneumoniae (about $60 \%$ carrier rate), implementation of the conjugated vaccine has tremendously decreased invasive pneumococcal diseases by vaccine serotypes not only in vaccinated but also in non-vaccinated individuals (due to herd immunity). However, the decrease in disease by vaccine 
serotypes has recently been accompanied by an increase of non-vaccine serotypes. This pre-placement of vaccine serotypes by non-vaccine serotypes is thought to be a consequence of selective pressure by the PCV-7. Most data on serotype replacement are from the US, where PCV-7 was introduced in 2000 [35]. In particular, the incidence of infections by frequently multiresistant isolates of serotype 19A, which has now been included in the extended PCV-13, has risen in the US within the last few years [36]. However, the role of PCV in replacement remains an issue of debate since countries that did not introduce PCV have also observed a rise in $19 \mathrm{~A}$ and historical surveillance studies from Denmark report an increase of 19A before the introduction of any pneumococcal vaccine [37].

The impact of the conjugated vaccine on non-invasive pneumococcal pneumonia is estimated be much higher. As for the PPV-23, however, data from randomized controlled trials are still missing. Currently, the placebocontrolled Community-Acquired Pneumonia Immunization Trial in Adults (CAPITA) is investigating the prevention of a first episode of vaccine serotype-specific pneumococcal CAP by the conjugated vaccine in 85,000 Dutch community-dwelling adults who are 65 years or older [38]. The results are pending.

Recently, extended conjugated vaccines with 10 or 13 serotypes have been licensed. Serotype 3 has been recognized not only as a frequent cause of CAP in adults but also as an independent risk factor for septic shock [39]. Therefore, it should be kept in mind that only the conjugated 13-valent and PPV-23 contain this important serotype.

Patients with severe chronic obstructive pulmonary disease (COPD) are at increased risk for exacerbations and pneumonia. Results on inhaled steroids are conflicting. Whereas inhaled steroids decrease the frequency of exacerbations, a large nested case control study within a cohort of COPD patients from Quebec $(175,906$ COPD patients, of whom 23,942 were hospitalized for pneumonia) demonstrated clearly that inhaled steroids increase the risk for pneumonia in a dose-dependent manner [40]. In particular, doses above an equivalent of fluticasone $1000 \mu \mathrm{g} /$ day seem to increase the risk relevantly.

In several epidemiological studies, smoking has been linked to increased susceptibility to CAP. A recent investigation uncovered one of the underlying mechanisms by demonstrating that smoking suppresses pulmonary innate host defense by impaired expression of antimicrobial peptides [41].

\section{Implications for clinical practice}

CAP remains a major public health threat. There are advances in treatment focusing on anti-inflammatory strategies. Some strategies can easily be transferred to daily practice (e.g., by using a combination therapy with macrolides or a respiratory fluoroquinolone with antiinflammatory activities instead of beta-lactam monotherapy in patients with more severe CAP).

However, currently, the prevention of pneumonia by vaccination seems to be the most promising field for real improvement. Since the performance of PPV-23 in this regard leaves room for improvement, much hope is based on the extended PCV and the results of the CAPITA study.

In the context of the current aH1N1 pandemic, it should be kept in mind that the majority of influenzaassociated pneumonia cases are not primary viral pneumonia but secondary bacterial pneumonias. In the context of the recent pandemic, several studies analyzing influenza pandemics of the last century have been published. S. pneumonia and, to a lesser extent, Haemophilus influenzae and Staphylococcus aureus were the leading bacterial causes of secondary pneumonia $[42,43]$. In this regard, the impact of combined influenza and pneumococcal vaccine cannot be overestimated.

\section{Abbreviations}

BALF, bronchoalveolar lavage fluid; BLM, beta-lactam/ macrolide; CAP, community-acquired pneumonia; CAPITA, Community-Acquired Pneumonia Immunization Trial in Adults; COPD, chronic obstructive pulmonary disease; CRB65, confusion, respiratory rate, blood pressure, and age (below or above 65 years) score; DPB, diffuse panbronchiolitis; ERK, extracellular signal-regulated kinase; IL, interleukin; LPS, lipoploysaccharide; NF-кB, nuclear factor-kappa-B; $\mathrm{PCV}$, pneumococcal conjugate vaccine; PPV-23, 23-valent pneumococcal polysaccharide vaccine; TNF- $\alpha$, tumor necrosis factor-alpha.

\section{Competing interests}

The authors declare that they have no competing interests.

\section{References}

I. Welte T, Kohnlein T: Global and local epidemiology of community-acquired pneumonia: the experience of the CAPNETZ Network. Semin Respir Crit Care Med 2009, 30:127-35.

2. Ruiz-Gonzalez A, Falguera M, Nogues A, Rubio-Caballero M: Is Streptococcus pneumoniae the leading cause of pneumonia of unknown etiology? A microbiologic study of lung aspirates in consecutive patients with community-acquired pneumonia. Am J Med 1999, 106:385-390.

3. Kudoh S: Erythromycin treatment in diffuse panbronchiolitis. Curr Opin Pulm Med 1998, 4:1 16-2I. 
4. Kudoh S, Azuma A, Yamamoto M, Izumi T, Ando M: Improvement of survival in patients with diffuse panbronchiolitis treated with low-dose erythromycin. Am J Respir Crit Care Med 1998, 157: 1829-32.

5. Shinkai M, Henke MO, Rubin BK: Macrolide antibiotics as immunomodulatory medications: proposed mechanisms of action. Pharmacol Ther 2008, I I 7:393-405.

6. Desaki M, Okazaki H, Sunazuka T, Omura S, Yamamoto K, Takizawa $\mathrm{H}$ : Molecular mechanisms of anti-inflammatory action of erythromycin in human bronchial epithelial cells: possible role in the signaling pathway that regulates nuclear factor-kappaB activation. Antimicrob Agents Chemother 2004, 48: $158 \mid-5$.

7. Shinkai M, Foster GH, Rubin BK: Macrolide antibiotics modulate ERK phosphorylation and IL-8 and GM-CSF production by human bronchial epithelial cells. Am J Physiol Lung Cell Mol Physiol 2006, 290:L75-85.

8. Bosnar M, Bosnjak B, Cuzic S, Hrvacic B, Marjanovic N, Glojnaric I, Culic O, Parnham MJ, Erakovic Haber V: Azithromycin and clarithromycin inhibit lipopolysaccharide-induced murine pulmonary neutrophilia mainly through effects on macrophage-derived granulocyte-macrophage colony-stimulating factor and interleukin-Ibeta. J Pharmacol Exp Ther 2009, 33I:104-13.

9. Uriarte SM, Molestina RE, Miller RD, Bernabo J, Farinati A, Eiguchi K, Ramirez JA, Summersgill JT: Effects of fluoroquinolones on the migration of human phagocytes through Chlamydia pneumoniae-infected and tumor necrosis factor alpha-stimulated endothelial cells. Antimicrob Agents Chemother 2004, 48:2538-43.

10. Riesbeck K: Immunomodulating activity of quinolones: review. J Chemother 2002, 14:3-12.

II. Weiss T, Shalit I, Blau H, Werber S, Halperin D, Levitov A, Fabian I: Anti-inflammatory effects of moxifloxacin on activated human monocytic cells: inhibition of NF-kappaB and mitogen-activated protein kinase activation and of synthesis of proinflammatory cytokines. Antimicrob Agents Chemother 2004, 48:1974-82.

12. Ogino H, Fujii M, Ono M, Maezawa K, Hori S, Kizu J: In vivo and in vitro effects of fluoroquinolones on lipopolysaccharideinduced pro-inflammatory cytokine production. J Infect Chemother 2009, 15:168-73.

13. Crosbie PA, Woodhead MA: Long-term macrolide therapy in chronic inflammatory airway diseases. Eur Respir J 2009, 33:|7|-8|.

14. Hardy RD, Rios AM, Chavez-Bueno S, Jafri HS, Hatfield J, Rogers BB, McCracken GH, Ramilo O: Antimicrobial and immunologic activities of clarithromycin in a murine model of Mycoplasma pneumoniae-induced pneumonia. Antimicrob Agents Chemother 2003, 47:1614-20.

15. Rios AM, Mejias A, Chavez-Bueno S, Fonseca-Aten M, Katz K, Hatfield J, Gomez AM, Jafri HS, McCracken GH Jr, Ramilo O, Hardy RD: Impact of cethromycin (ABT-773) therapy on microbiological, histologic, immunologic, and respiratory indices in a murine model of Mycoplasma pneumoniae lower respiratory infection. Antimicrob Agents Chemother 2004, 48:2897-904.

16. Tormakangas L, Saario E, Bem David D, Bryskier A, Leinonen M, Saikku P: Treatment of acute Chlamydia pneumoniae infection with telithromycin in C57BL/6J mice. J Antimicrob Chemother 2004, 53:1101-4.

17. Martinez JA, Horcajada JP, Almela M, Marco F, Soriano A, Garcia E, Marco MA, Torres A, Mensa J: Addition of a macrolide to a betalactam-based empirical antibiotic regimen is associated with lower in-hospital mortality for patients with bacteremic pneumococcal pneumonia. Clin Infect Dis 2003, 36:389-95.

18. Garcia Vazquez E, Mensa J, Martinez JA, Marcos MA, Puig J, Ortega M, Torres A: Lower mortality among patients with communityacquired pneumonia treated with a macrolide plus a betalactam agent versus a beta-lactam agent alone. Eur J Clin Microbiol Infect Dis 2005, 24:190-5.
19. Metersky ML, Ma A, Houck PM, Bratzler DW: Antibiotics for bacteremic pneumonia: Improved outcomes with macrolides but not fluoroquinolones. Chest 2007, I 3 1:466-73.

FI000 Factor 6.0 Must Read

Evaluated by Richard Wunderink 10 Apr 2007

20. Restrepo MI, Mortensen EM, Waterer GW, Wunderink RG, Coalson JJ, Anzueto A: Impact of macrolide therapy on mortality for patients with severe sepsis due to pneumonia. Eur Respir J 2009, 33:153-9.

FI000 Factor 4.9 Must Read

Evaluated by Mathias Pletz 26 Jan 2009, Klaus Dalhoff 20 Feb 2009, Charles Feldman II May 2009

21. Tessmer A, Welte T, Martus P, Schnoor M, Marre R, Suttorp N: Impact of intravenous $\{$ beta\}-lactam/macrolide versus $\{$ beta $\}$ lactam monotherapy on mortality in hospitalized patients with community-acquired pneumonia. J Antimicrob Chemother 2009, 63:1025-33.

22. Finch $R$, Schurmann $D$, Collins $O$, Kubin R, McGivern J, Bobbaers $H$, Izquierdo JL, Nikolaides P, Ogundare F, Raz R, Zuck P, Hoeffken G: Randomized controlled trial of sequential intravenous (i.v.) and oral moxifloxacin compared with sequential i.v. and oral co-amoxiclav with or without clarithromycin in patients with community-acquired pneumonia requiring initial parenteral treatment. Antimicrob Agents Chemother 2002, 46: 1746-54.

23. Chalmers JD, Singanayagam A, Murray MP, Hill AT: Prior statin use is associated with improved outcomes in communityacquired pneumonia. Am J Med 2008, I2I:|002-1007.el.

FI000 Factor 3.0 Recommended Evaluated by Charles Feldman 10 Feb 2009

24. Dublin S, Jackson ML, Nelson JC, Weiss NS, Larson EB, Jackson LA: Statin use and risk of community acquired pneumonia in older people: population based case-control study. BMJ 2009, 338:b2। 37

25. Glynn RJ, Schneeweiss S, Wang PS, Levin R, Avorn J: Selective prescribing led to overestimation of the benefits of lipidlowering drugs. J Clin Epidemiol 2006, 59:8|9-28.

26. Marik PE, Pastores SM, Annane D, Meduri GU, Sprung CL, Arlt W, Keh D, Briegel J, Beishuizen A, Dimopoulou I, Tsagarakis S, Singer M, Chrousos GP, Zaloga G, Bokhari F, Vogeser M: Recommendations for the diagnosis and management of corticosteroid insufficiency in critically ill adult patients: consensus statements from an international task force by the American College of Critical Care Medicine. Crit Care Med 2008, 36:1937-49.

27. Confalonieri M, Urbino R, Potena A, Piattella M, Parigi P, Puccio G, Della Porta R, Giorgio C, Blasi F, Umberger R, Meduri GU: Hydrocortisone infusion for severe community-acquired pneumonia: a preliminary randomized study. Am J Respir Crit Care Med 2005, 171:242-8.

28. Garcia-Vidal C, Calbo E, Pascual V, Ferrer C, Quintana S, Garau J: Effects of systemic steroids in patients with severe community-acquired pneumonia. Eur Respir J 2007, 30:95I-6.

FI000 Factor 3.0 Recommended Evaluated by Charles Feldman 22 Oct 2007

29. Snijders D, Daniels JM, de Graaff CS, van der Werf TS, Boersma WG: Efficacy of corticosteroids in community-acquired pneumonia: a randomized double-blinded clinical trial. Am J Respir Crit Care Med 2010, 181:975-82

FI000 Factor 3.2 Recommended

Evaluated by Shawn Skerrett 0 I Mar 2010, Jadwiga Wedzicha I 4 Jun 2010

30. Gorman SK, Slavik RS, Marin J: Corticosteroid treatment of severe community-acquired pneumonia. Ann Pharmacother 2007, 4I:1233-7.

31. Moberley SA, Holden J, Tatham DP, Andrews RM: Vaccines for preventing pneumococcal infection in adults. Cochrane Database Syst Rev 2008, I:CD000422. 
32. Maruyama T, Taguchi $\mathrm{O}$, Niederman MS, Morser J, Kobayashi $\mathrm{H}$, Kobayashi T, D'Alessandro-Gabazza C, Nakayama S, Nishikubo K, Noguchi T, Takei Y, Gabazza EC: Efficacy of 23-valent pneumococcal vaccine in preventing pneumonia and improving survival in nursing home residents: double blind, randomised and placebo controlled trial. BMJ 2010,340:c1004.

33. Teshale EH, Hanson D, Flannery B, Phares C, Wolfe M, Schuchat A, Sullivan P: Effectiveness of 23-valent polysaccharide pneumococcal vaccine on pneumonia in HIV-infected adults in the United States, 1998-2003. Vaccine 2008, 26:5830-4.

FI000 Factor 3.0 Recommended

Evaluated by Laurence Huang 30 Jan 2009

34. Pletz MW, Maus U, Krug N, Welte T, Lode H: Pneumococcal vaccines: mechanism of action, impact on epidemiology and adaption of the species. Int J Antimicrob Agents 2008, 32:199-206.

35. Moore MR, Whitney CG: Emergence of nonvaccine serotypes following introduction of pneumococcal conjugate vaccine: cause and effect? Clin Infect Dis 2008, 46:183-5.

36. Moore MR, Gertz RE Jr, Woodbury RL, Barkocy-Gallagher GA, Schaffner W, Lexau C, Gershman K, Reingold A, Farley M, Harrison LH, Hadler JL, Bennett NM, Thomas AR, McGee L, Pilishvili T, Brueggemann AB, Whitney CG, Jorgensen JH, Beall $B$ : Population snapshot of emergent Streptococcus pneumoniae serotype 19A in the united states, 2005. J Infect Dis 2008, 197:1016-27.

37. Harboe ZB, Benfield TL, Valentiner-Branth P, Hjuler T, Lambertsen L, Kaltoft M, Krogfelt K, Slotved HC, Christensen JJ, Konradsen HB: Temporal trends in invasive pneumococcal disease and pneumococcal serotypes over 7 decades. Clin Infect Dis 2010, 50:329-37.

38. Hak E, Grobbee DE, Sanders EA, Verheij T], Bolkenbaas M, Huijts SM, Gruber WC, Tansey S, McDonough A, Thoma B, Patterson S, van Alphen AJ, Bonten MJ: Rationale and design of CAPITA: a RCT of I3-valent conjugated pneumococcal vaccine efficacy among older adults. Neth J Med 2008, 66:378-83.
39. Garcia-Vidal C, Ardanuy C, Tubau F, Viasus D, Dorca J, Linares J, Gudiol F, Carratala J: Pneumococcal pneumonia presenting with septic shock: host- and pathogen-related factors and outcomes. Thorax 2010, 65:77-81.

FI000 Factor 6.5 Must Read

Evaluated by Terence Seemungal 09 Feb 2010, Charles Feldman 16 Feb 2010, Klaus Dalhoff 26 Apr 2010

40. Ernst $P$, Gonzalez $A V$, Brassard $P$, Suissa S: Inhaled corticosteroid use in chronic obstructive pulmonary disease and the risk of hospitalization for pneumonia. Am J Respir Crit Care Med 2007, 176:162-6.

\section{Changes Clinical Practice}

FI000 Factor 3.2 Recommended

Evaluated by Antoni Torres 15 Aug 2007, Robin D Taylor 29 Aug 2007

4I. Herr C, Beisswenger C, Hess C, Kandler K, Suttorp N, Welte T, Schroeder JM, Vogelmeier C: Suppression of pulmonary innate host defence in smokers. Thorax 2009, 64: I44-9.

FI000 Factor 6.0 Must Read

Evaluated by David A Groneberg 14 Jan 2009

42. Gupta RK, George R, Nguyen-Van-Tam JS: Bacterial pneumonia and pandemic influenza planning. Emerg Infect Dis 2008, 14: I 187-92.

FI000 Factor 6.0 Must Read Evaluated by Mathias Pletz 18 Jun 2009

43. Morens DM, Taubenberger JK, Fauci AS: Predominant role of bacterial pneumonia as a cause of death in pandemic influenza: implications for pandemic influenza preparedness. $J$ Infect Dis 2008, 198:962-70.

FI000 Factor 4.8 Must Read

Evaluated by Joseph Mizgerd 28 Aug 2008, Ralph Corey II Nov 2008 